\title{
Editorial
}

\author{
Callum G. Fraser
}

\section{Assuring the quality of examinations using faecal immunochemical tests for haemoglobin (FIT)}

https://doi.org/10.1515/cclm-2020-1509

Keywords: accreditation; biological variation; colorectal cancer; faecal tests; laboratory; quality assurance.

Faecal immunochemical tests for haemoglobin (FIT) are now regarded as the best non-invasive investigation for screening asymptomatic individuals for the presence of colorectal cancer (CRC) [1]. They are now used in opportunistic and programmatic screening all over the world. FIT are also used in surveillance programmes for patients following removal of polyps [2]. In addition, the evidence has grown rapidly over recent years that FIT, in conjunction with symptoms and clinical judgement, provide a very useful tool in deciding which patients presenting in primary care with lower bowel symptoms will benefit most from further investigation, usually bowel visualisation [3].

Although FIT are available as qualitative and quantitative examinations [4], the latter give numerical estimates of faecal haemoglobin concentrations (f- $\mathrm{Hb})$. These are now favoured in all three of the clinical settings in which FIT are of value This is especially relevant in the current COVID-19 pandemic, in which $\mathrm{f}-\mathrm{Hb}$ have become widely recommended by governments, as well as professional bodies and local health organisations, for use in prioritising the further investigation of participants in screening and patients presenting with symptoms, when they have $\mathrm{f}-\mathrm{Hb}$ greater than the threshold $\mathrm{f}-\mathrm{Hb}$ used to recommend referral [5]. Numerical threshold $\mathrm{f}-\mathrm{Hb}$ are used, usually nationally or at least regionally, for the interpretation of FIT results in all clinical settings, particularly since population-based reference values are inappropriate [6]. In consequence, good examination quality and rigorous performance of examinations are both required. The International Organization for Standardization (ISO)

Professor Callum G. Fraser, Centre for Research into Cancer Prevention and Screening, University of Dundee, Ninewells Hospital and Medical School, Dundee DD1 9SY, Scotland, UK, E-mail: c.g.fraser@dundee.ac.uk.https://orcid.org/0000-00021333-7994
15189:2012 specifies requirements for quality and competence in medical laboratories [7].

\section{Internal quality control}

ISO 15189, section 5.6.2.2, states: Quality control materials: The laboratory shall use quality control materials that react to the examining system in a manner as close as possible to patient samples. It is noted that the laboratory should choose concentrations of control materials, wherever possible, especially at or near clinical decision values, which ensure the validity of decisions made. Use of independent third party control materials should be considered, either instead of, or in addition to, any control materials supplied by the reagent or instrument manufacturer. However, despite these laudable ideals, haemoglobin $(\mathrm{Hb})$ in faeces is unstable and faecal based matrices for internal quality control (IQC) materials are probably unrealistic. In addition, it has been recently noted that third party ICQ materials are lacking [3].

A possible strategy to overcome this latter deficiency has now been examined [8]. The study assessed whether the two or three IQC materials available from each of the four manufacturers of quantitative FIT systems, marketed in the United Kingdom (UK) for ongoing evaluation of their own systems, were compatible with the other three systems. This was achieved by quantitative estimation of within-run and between-run imprecision. Interestingly, all results were reported without flagged errors on all the FIT systems, which supports the concept that transferability of the IQC materials between systems is indeed possible. This seems logical given that these IQC materials are either liquid or reconstituted lyophilised $\mathrm{Hb}$ solutions. There is an obvious limitation to this strategy in that the mean $\mathrm{f}-\mathrm{Hb}$ found on examinations of each IQC material were different from those originally assigned by each manufacturer for their own FIT system. However, this is unsurprising given that there is currently no international standardisation or harmonisation of FIT, the methods are based on polyclonal antibodies, use different calibration techniques, are traceable to different primary reference materials and do not have identical 
examination performance characteristics [9]. Despite this, it was concluded that, if manufacturers were willing to market their IQC materials independently of their FIT systems, the potential exists for third party IQC materials for FIT to be widely available.

\section{External quality assessment}

ISO 15189, section 5.6.3.1 states: The laboratory shall participate in an interlaboratory comparison programme(s) (such as an external quality assessment [EQAS] programme or proficiency testing programme) appropriate to the examination and interpretations of examination results. The laboratory shall monitor the results of the interlaboratory comparison programme(s) and participate in the implementation of corrective actions when predetermined performance criteria are not fulfilled. However, following a review of the existing challenges and constraints, Godber et al. did state that the organisers of EQAS for FIT have many difficulties in providing appropriate materials [3].

In addition to assessing transferability of IQC materials across FIT systems discussed above [8] and comparing the examination performance characteristics of four FIT systems [9], the research group have studied this aspect of the quality assurance of quantitative FIT. The rationale for the study was that, since EQAS are being established worldwide to support FIT examinations, the suitability of the materials currently provided by commercially available EQAS should be assessed [10]. Further, the EQAS challenges available for FIT do come in a variety of formats, each unique to the individual EQAS, which might make it difficult for medical laboratories to decide in which one or more EQAS to participate. For the study, EQAS organisers provided one or more materials with the following matrices: $\mathrm{Hb}$ spiked artificial faecal like matrix sent to participating laboratories for loading into FIT specimen collection devices, $\mathrm{Hb}$ spiked artificial faecal like matrix pre-loaded into FIT specimen collection devices by the EQAS organisers and then sent to participating laboratories, diluted $\mathrm{Hb}$ directly injected into the appropriate FIT system specimen collection devices by the EQAS organisers, $\mathrm{Hb}$ spiked liquid provided either lyophilised for reconstitution by the participating medical laboratory, or liquid ready to use after being portioned into system cups for direct sampling on the FIT system. The examinations were performed on the same four FIT systems used in the study of IQC materials [8] and previously evaluated [9]. The imprecision found on examination of samples with a faecal like matrix had a higher median CV (12.4-19.0\%) when compared to those from samples with liquid matrices
(0.8-2.3\%). The range of CV was also higher for samples from faecal like matrix EQAS compared to liquid samples.

Although samples from participants in screening and patients with symptoms arrive in the medical laboratory after collection into the appropriate specimen collection devices, it was the opinion of the authors that it is not necessary for EQAS materials to be provided as a faecal like matrix. The rationale was that faecal like matrices, whilst clearly more consistent with participant or patient samples, are prone to pre-examination variation and so do not assess the examination performance characteristics of a FIT system. Moreover, a problem with samples with faecal like matrices is that it is unclear whether any differences observed from the target values are due to pre-examination or examination variation. Samples with liquid matrices are not prone to preexamination variation and were considered better able to assess the examination performance of a FIT system.

\section{Discussion}

It has been shown that the use of IQC materials from one FIT system manufacturer as third party controls for other systems seems feasible [8]. However, several questions remain unresolved. The major issue is that, at present, medical laboratories would have to assign the $\mathrm{f}-\mathrm{Hb}$ for the particular FIT system used and set the criteria for acceptance or rejection of analyses. Leading third party IQC material manufacturers assign target values using data from independent laboratories. If independent target $\mathrm{f}-\mathrm{Hb}$ data were unavailable for the FIT system used, then the IQC material cannot really be termed third party. Would manufacturers of FIT systems procure assignment of target values for their own IQC materials for other manufacturers' systems using independent laboratories? Although available FIT systems give different results, are the differences consistent over the measurement ranges of the systems so that factors could be applied to assign target values by simple multiplication of the manufacturer assigned $\mathrm{f}-\mathrm{Hb}$ ? Would such factors be consistent over different lots of the critical antibody reagent? Could the target $\mathrm{f}-\mathrm{Hb}$, appropriate to the decision threshold, be modified by diluting liquid IQC materials, or using different volumes of diluent for lyophilised IQC materials? Further clarification of these issues seems warranted.

The application of currently available EQAS across four FIT systems was also investigated [10]. Results of EQAS challenges usually give data on comparative bias between systems since the traditional view is that, while IQC monitors the imprecision of examinations (and also gives information on sudden or gradual shifts in performance), EQAS should be providing an assessment of bias 
with respect to the same and other methods. However, the authors decided, due to the lack of a suitable reference material and standardisation of FIT methods, to perform this study on imprecision. The differences between the $\mathrm{f}-\mathrm{Hb}$ found using different FIT systems requires further study and documentation. The results from EQAS could greatly assist in clarification of this important facet of FIT.

The publications discussed here $[8,10]$ provide further interesting insights to improve the state-of-the-art of testing for the presence of $\mathrm{Hb}$ in faeces: further studies are required to continue this work. Thankfully, the Working Group on FIT of the Scientific Division of the International Federation of Clinical Chemistry and Laboratory Medicine are actively pursuing strategies to improve the harmonisation of FIT systems.

Research funding: None declared.

Author contributions: The sole author has accepted responsibility for the entire content of this manuscript and approved its submission.

Competing interests: The author undertakes paid consultancy for Hitachi Chemical Diagnostic Systems Co. Ltd, Tokyo, Japan.

\section{References}

1. Robertson DJ, Selby K. Fecal immunochemical test: the world's colorectal cancer screening test. Gastrointest Endosc Clin N Am 2020;30:511-26.
2. Young GP, Woodman RJ, Symonds E. Detection of advanced colorectal neoplasia and relative colonoscopy workloads using quantitative faecal immunochemical tests: an observational study exploring the effects of simultaneous adjustment of both sample number and test positivity threshold. BMJ Open Gastroenterol 2020;7:e000517.

3. Godber IM, Benton SC, Fraser CG. Setting up a service for a faecal immunochemical test for haemoglobin (FIT): a review of considerations, challenges and constraints. J Clin Pathol 2018;71: 1041-5.

4. Allison JE, Fraser CG, Halloran SP, Young GP. Population screening for colorectal cancer means getting FIT: the past, present, and future of colorectal cancer screening using the fecal immunochemical test for hemoglobin (FIT). Gut Liver 2014;8:117-30.

5. D'Souza N, Abulafi M. Navigating the storm of COVID-19 for patients with suspected bowel cancer. Br J Surg 2020;107:e204.

6. Clark GRC, Strachan JA, McPherson A, Digby J, Mowat C, Steele RJC et al. Faecal haemoglobin distributions by sex, age, deprivation and geographical region: consequences for colorectal cancer screening strategies. Clin Chem Lab Med 2020;58:2073-80.

7. International Organization for Standardization. ISO. Medical laboratories - Requirements for quality and competence (ISO 15189); 2012.

8. Piggott C, Shugaa Z, Benton SC. Independent internal quality control (IQC) for faecal immunochemical tests (FIT) for haemoglobin: use of FIT manufacturers' IQC for other FIT systems. Clin Chem Lab Med 2021;59:e41-3.

9. Piggott C, Carroll MRR, John C, O’Driscoll S, Benton SC. Analytical evaluation of four faecal immunochemistry tests for haemoglobin. Clin Chem Lab Med 2021;59:173-8.

10. O'Driscoll S, Piggott C, Bruce H, Benton SC. An evaluation of ten external quality assurance scheme (EQAS) materials for the faecal immunochemical test (FIT) for haemoglobin. Clin Chem Lab Med 2021;59:307-13. 